\title{
Critical behavior of the three-dimensional bond-diluted Ising spin glass: Finite-size scaling functions and universality
}

\author{
Thomas Jörg ${ }^{1}$ \\ ${ }^{1}$ Dipartimento di Fisica, Università di Roma "La Sapienza", \\ SMC and INFM, P.le. Aldo Moro 2, 00185 Roma, Italy.
}

(Dated: June 28, 2018)

\begin{abstract}
We study the three-dimensional (3D) bond-diluted Edwards-Anderson (EA) model with binary interactions at a bond occupation of $45 \%$ by Monte Carlo (MC) simulations. Using an efficient cluster MC algorithm we are able to determine the universal finite-size scaling (FSS) functions and the critical exponents with high statistical accuracy. We observe small corrections to scaling for the measured observables. The critical quantities and the FSS functions indicate clearly that the bond-diluted model for dilutions above the critical dilution $p^{*}$, at which a spin glass (SG) phase appears, lies in the same universality class as the 3D undiluted EA model with binary interactions. A comparison with the FSS functions of the 3D site-diluted EA model with Gaussian interactions at a site occupation of $62.5 \%$ gives very strong evidence for the universality of the SG transition in the 3D EA model.
\end{abstract}

PACS numbers: $75.10 . \mathrm{Nr}$

\section{INTRODUCTION}

Almost all the Monte Carlo (MC) simulations of the three-dimensional (3D) Edwards-Anderson (EA) model ${ }^{1}$ have been performed using either an undiluted Gaussian or binary coupling distribution $\stackrel{2}{2}$ There are, however, many reasons to study the 3D bond-diluted EA model with binary interactions. The simplest reason being that it allows one, although in a very crude way, to approximate the presence of weak bonds in the model with Gaussian couplings without loosing the technical benefits of the binary couplings. The introduction of vacant bonds reduces the frustration in the model, making it easier to find ground states, which should facilitate low-temperature MC simulations. Moreover, the bonddiluted model in a certain range of the dilution allows for an efficient use of replica cluster MC algorithms $\stackrel{3,4,5,6,7}{\text { (n) }}$ It has been shown that the high-temperature series expansion typically converges better for some intermediate dilution than for the undiluted case. $\stackrel{8}{=}$ Similarly, it was found that the scaling of the ground state defect energies is much improved at intermediate dilutions in comparison to the standard undiluted case, that showed to scale rather poorly $\underline{\underline{9}}$ This gives some evidence that in general the scaling violations of the diluted model, for some intermediate range of the dilution, might be smaller than in the undiluted case. This fact is very important for spin glass (SG) simulations since the range of system sizes that are accessible to $\mathrm{MC}$ calculations is very limited due to the presence of large free energy barriers in the SG phase, causing slow thermalization. The problems of the very restricted simulation range are worsened by the fact that the leading correction-to-scaling exponent $\omega$ has been measured to be probably smaller than one, 10,11 such that the leading corrections fall off only slowly. All this combined with the need of excellent statistics makes a reliable estimate of critical exponents very difficult. In addition to these rather technical aspects the bond-diluted $\pm J$ EA model may be thought of simply as an EA model with a particular distribution of the couplings and therefore it also serves to provide insights on the debated issue of the universality of the 3D EA model $12,13,14,15,16,17,18$

In this paper we first consider in detail the bonddiluted 3D EA model at a bond occupation of $45 \%$ which is close to the value where optimal scaling for the

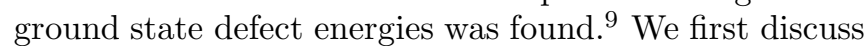
the replica cluster MC algorithm we use and its performance, then we focus on the SG transition, the corresponding critical exponents and the universal finite-size scaling (FSS) functions of the correlation length $\xi$, the SG susceptibility $\chi_{\mathrm{SG}}$ and the Binder ratio $g$. We determine these FSS functions with very high statistical accuracy (up to $10^{5}$ samples per lattice size) and use them to determine critical exponents with two different methods, namely, the quotient method ${ }^{10,19}$ and an extrapolation to infinite volume method $\stackrel{20}{\underline{w}}$ We find excellent agreement between the critical exponents extracted by these two different methods, as well as with the values given by the high-precision study of the undiluted binary model of Ballesteros et al $\underline{\underline{10}}$ In addition, we find that the FSS functions agree very well with the ones obtained by Palassini and Caracciolo for the undiluted binary model $\stackrel{11}{\underline{1}}$ This together gives strong evidence that the SG transition of the 3D bond-diluted EA model with binary couplings falls into the same universality class as the one of the corresponding undiluted model, as indicated by high-temperature expansion ${ }^{8}$ and as one might expect on general grounds $\stackrel{21}{=}$ We then compare the FSS functions to the ones of the 3D site-diluted EA model at a site occupation of $62.5 \%$ with a Gaussian distribution of the couplings $\underline{\underline{7}}$ In contrast to the bond-diluted $\pm J$ model the site-diluted Gaussian model shows very noticeable finite-size effects and therefore the agreement between the FSS functions of the two models emerges only at large enough sizes. Despite the fact that the two models have finite-size effects of clearly different magni- 
tude the agreement between the FSS functions gets excellent asymptotically. This leads us to the conclusion that the critical behavior of the $3 \mathrm{D}$ EA model is most probably universal and that violations of universality as they are found in dynamical MC simulations ${ }^{12,13,14,16}$ are probably due to difficulties to control the corrections to scaling in such simulations. ${ }^{22,23}$ In Ref. 17 the conclusion that the critical behavior of the $3 \mathrm{D}$ EA model is universal is reached from a high-precision MC study in which the static properties of the model with Gaussian and $\pm J$ couplings are compared.

We find indications that scaling violations at the given bond occupation are in fact reduced in comparison to the standard undiluted model, making the study of the bond-diluted $\pm J$ EA very interesting for various reasons.

Finally, note that all the figures in this paper where the coupling distribution is not explicitly mentioned in the caption refer to the data obtained from the study of the bond-diluted $\pm J$ EA model.

\section{THE MODELS}

\section{A. Bond-diluted $\pm J$ EA model}

We mainly consider the 3D bond-diluted EA model given by the Hamiltonian 1

$$
\mathcal{H}=-\sum_{\langle x y\rangle} J_{x y} \epsilon_{x y} \sigma_{x} \sigma_{y}
$$

where the sum is over all the nearest neighbor pairs of a simple cubic 3D lattice of length $L$. The $\sigma_{x}= \pm 1$ are Ising spins and the $\epsilon_{x y}$ are the bond occupation variables, i.e., $\epsilon_{x y}=0$ for an empty and $\epsilon_{x y}=1$ for an occupied bond. For the couplings $J_{x y}$ we use a binary distribution, i.e., $J_{x y}= \pm 1$. The fraction of occupied bonds $p$ in this study is $p=0.45$. We use periodic boundary conditions to study the Hamiltonian in Eq. (11). Note that a SG transition occurs only at and above a certain critical dilution $p^{*}$ that is found to be slightly larger than the bond percolation threshold $p_{c}=0.2488$ for discrete distributions of the couplings. 9.24 This is in contrast to the case of continuous coupling distributions, where the SG phase appears exactly at $p_{c}$. A detailed discussion on the value of $p^{*}$ will be given in Ref. 23 .

\section{B. Site-diluted Gaussian EA model}

In order to compare the FSS functions for two different coupling distributions we also consider the 3D sitediluted EA model with a Gaussian coupling distribution given by the Hamiltonian 1

$$
\mathcal{H}=-\sum_{\langle x y\rangle} J_{x y} \epsilon_{x} \epsilon_{y} \sigma_{x} \sigma_{y}
$$

where the sum is again over all the nearest neighbor pairs of a simple cubic 3D lattice of length $L$. The $\sigma_{x}= \pm 1$ are Ising spins and the $\epsilon_{x}$ are the site occupation variables, i.e., $\epsilon_{x}=0$ for an empty and $\epsilon_{x}=1$ for an occupied site. For the couplings $J_{x y}$ we use a Gaussian distribution with mean zero and variance unity. The fraction of occupied sites $r$ in this study is $r=0.625$. For this model the SG phase is supposed to be present above the site percolation threshold $r_{c}=0.3116$. We again use periodic boundary conditions to study the Hamiltonian in Eq. (2).

\section{THE FSS METHOD}

Taking a suitably defined finite-volume correlation length $\xi(T, L)$ and a long-range observable $\mathcal{O}(T, L)$ as, e.g., $\chi_{\mathrm{SG}}(T, L)$ FSS theory predicts ${ }^{25,26}$

$$
\frac{\mathcal{O}(T, L)}{\mathcal{O}(T, \infty)}=f_{\mathcal{O}}[\xi(T, \infty) / L]+O\left(\xi^{-\omega}, L^{-\omega}\right)
$$

and

$$
\frac{\mathcal{O}(T, s L)}{\mathcal{O}(T, L)}=F_{\mathcal{O}}[\xi(T, L) / L ; s]+O\left(\xi^{-\omega}, L^{-\omega}\right)
$$

where $f_{\mathcal{O}}$ and $F_{\mathcal{O}}$ are universal FSS functions and $s>1$ is a scale factor. Eq. (4) is an excellent starting point for numerical investigations of the FSS behavior, as it involves only finite-volume quantities taken from a pair of systems with sizes $L$ and $s L$ at a given temperature $T$. The knowledge of the universal scaling functions $F_{\mathcal{O}}$ and $F_{\xi}$ allows us to extract critical exponents either by infinite volume extrapolation ${ }^{11,20}$ or by the quotient method, 10,19 For the quotient method one defines an effective critical temperature $T_{c}^{*}$ at which the correlation length measured in units of the lattice size $L$ are equal for the pair of systems, i.e.,

$$
\xi\left(T_{c}^{*}, L\right) / L=\xi\left(T_{c}^{*}, s L\right) /(s L) .
$$

For an observable $\mathcal{O}$ that in the thermodynamic limit diverges as $t^{-x_{\mathcal{O}}}\left(t=T / T_{c}-1\right.$ being the reduced temperature) the critical exponent $x_{\mathcal{O}}$ can be extracted from the quotient

$$
s^{x_{\mathcal{O}} / \nu}=\frac{\mathcal{O}\left(T_{c}^{*}, s L\right)}{\mathcal{O}\left(T_{c}^{*}, L\right)}+\mathcal{O}\left(L^{-\omega}\right)
$$

and hence from $F_{\mathcal{O}}$. The critical exponent $\nu$ describing the divergence of $\xi$ can be, e.g., measured from the FSS function of the temperature derivative of $\xi$, i.e., from $F_{\partial_{\mathrm{T}} \xi}$, which after a short calculation leads to

$$
s^{1 / \nu}=1+\left.\frac{x^{*}}{s} \partial_{x} F_{\xi}(x ; s)\right|_{x=x^{*}}+O\left(L^{-\omega}\right)
$$

with $x=\xi(T, L) / L$ and $x^{*}=\xi\left(T_{c}^{*}, L\right) / L$. The infinite volume extrapolation technique works with data strictly above $T_{c}$ and uses the FSS functions $F_{\xi}$ and $F_{\mathcal{O}}$ to obtain the thermodynamic limit of $\mathcal{O}$ using an iterative 
procedure in which the pair $\xi$ and $\mathcal{O}$ is scaled up from $L \rightarrow s L \rightarrow s^{2} L \rightarrow \ldots \rightarrow \infty$ as described in detail in Ref. 20. Assuming an ordinary second-order phase transition $x_{\mathcal{O}}$ can be extracted from a power-law fit to the extrapolated data of $\mathcal{O}(T, \infty)$.

\section{ALGORITHM AND COMPUTATIONAL DETAILS}

In this MC study we make use of the fact that the Hamiltonians in Eqs. (11) and (2) allow for an efficient use of replica cluster algorithms that were originally used for the study of the two-dimensional EA model 3,5,6 The details of the algorithm as they are given below apply to the case of the bond-diluted $\pm J$ model, whereas a discussion of the algorithm in the case of the site-diluted Gaussian case can be found in Ref. 7. The basic idea of all these algorithms is that two independent replicas of the system are simulated simultaneously. Hence each lattice site can be associated with two spins, one from each replica, and can be in one of the four spin states $(++),(+-),(-+)$, and $(--)$. The $(+-)$ and $(-+)$ sites are called A sites and the $(++)$ and $(--)$ sites B sites. On these two different groups of sites it is now possible to define clusters in various ways. In the following we define the cluster moves used for this simulation. First we consider the clusters on the A sites. Following Redner et $a l^{\underline{4}}$ we grow the clusters by adding links between two A sites with probability $p_{a d d}$ given by

$$
p_{\text {add }}=\left\{\begin{array}{l}
1-\exp \left(4 \beta E_{x y}\right) \quad \text { if } \quad E_{x y}<0 \\
0 \quad \text { else }
\end{array}\right.
$$

where $E_{x y}=-J_{x y} \epsilon_{x y} \sigma_{x} \sigma_{y}$ is the energy contribution of the given link and $\beta$ is the inverse temperature. We repeat the same procedure for the links between the $B$ sites. Finally, we flip each of the clusters that are defined through this procedure with probability $1 / 2$, i.e., we make a Swendsen-Wang update. ${ }^{27}$ This choice is influenced by the findings that even in a diluted ferromagnet an update of all clusters can be efficient. ${ }^{28}$ In the presence of an external field the algorithm would have to be modified slightly as in this case only the clusters on the A sites flip freely $\underline{\underline{4}, 5}$

In order to improve the performance of this cluster algorithm we also update each replica with parallel tempering (PT) (Ref. 29) as proposed by Houdayer $\frac{5}{-}$ This means that in addition to the two replicas at temperature $T$ we introduce replicas on a set of $N_{T}$ temperatures $\left(T_{i}\right.$ with $\left.i=1, \ldots, N_{T}\right)$ and allow two replicas at $T_{i}$ and $T_{i+1}$ to exchange their temperatures with the appropriate probability. ${ }^{29}$ The resulting algorithm is only a slight variation of the original replica cluster algorithm by Swendsen and Wang. $\frac{3}{-}$ In contrast to Houdayer's algorithm ${ }^{5}$ it does not need PT to ensure ergodicity, because the different definition of the clusters already provides for it. The dilution in the coupling distribution

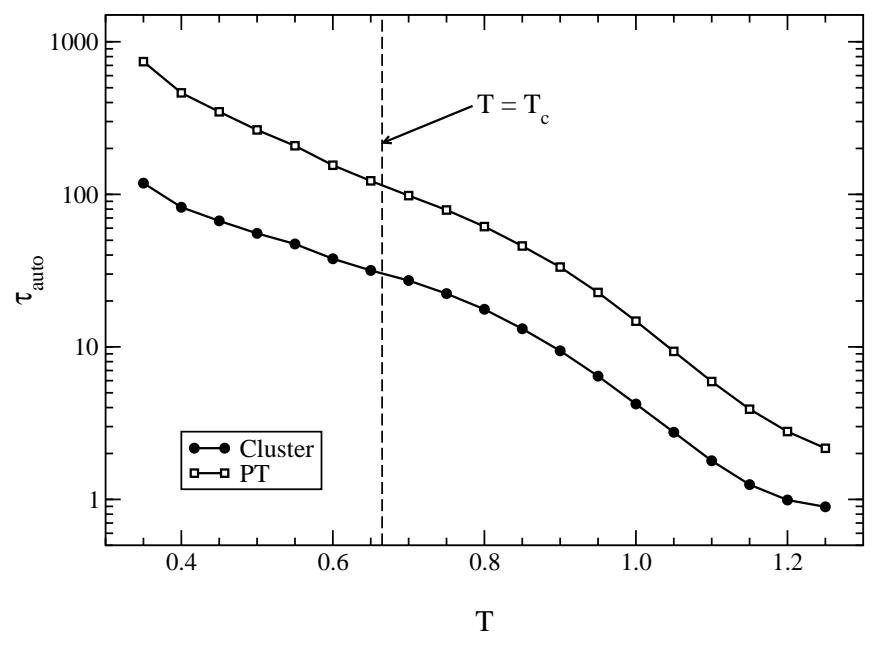

FIG. 1: Qualitative comparison of the integrated autocorrelation time $\tau_{\text {auto }}$ as a function of temperature between parallel tempering (PT) and the replica cluster algorithm (Cluster) averaged on the same 20 configurations at $L=10$.

is very important in order to avoid the site percolation problem mentioned by Houdayer.$^{\frac{5}{6}}$ Using, e.g., a nondiluted $\pm J$ coupling distribution the cluster move essentially just swaps the two replicas. That dilution may help greatly to speed up cluster algorithms in SG simulations has already been noted in the case of the Viana-Bray mode $1^{30,31}$. Finally, we note that it would also be possible to define clusters along the lines of Ref. 32, where the size of the resulting clusters can be controlled in a more general manner.

An important technical advantage of this algorithm with the given discrete distribution of the couplings is that it can be completely multispin coded, apart from the task where the clusters are identified with the HoshenKopelman algorithm. ${ }^{33}$ In Fig. 1 we compare the integrated autocorrelation times of the square of the order parameter $q^{2}$ (as defined below) of this cluster algorithm with the corresponding ones of PT with the same number of replicas simulated in total, i.e., with the same number of temperature slices, the same set of temperatures, and two replicas at each temperature. For this qualitative comparison we show an average over the same 20 configurations on $L=10$ lattices. One sees that in the SG phase the autocorrelation time of the cluster algorithm for the particular choice of temperatures and size is one order of magnitude smaller than the one of PT. The computational overhead of the cluster move can be kept rather small, such that the overall performance remains clearly favorable for the cluster algorithm.

We measure the overlap $q_{x}=\sigma_{x} \tau_{x}$ and $q=L^{-3} \sum_{x} q_{x}$ from two replicas $\sigma$ and $\tau$ with the same couplings $J_{x y} \epsilon_{x y}$. We define $\xi(T, L)$ by the second-moment correlation length $10,11,20,34,35$

$$
\xi(T, L)=\frac{1}{2 \sin \left(\left|\mathbf{k}_{\min }\right| / 2\right)}\left[\frac{\chi_{\mathrm{SG}}(\mathbf{0})}{\chi_{\mathrm{SG}}\left(\mathbf{k}_{\min }\right)}-1\right]^{\frac{1}{2}},
$$




\begin{tabular}{rrrrc}
\hline \hline$L$ & $N_{s}$ & $N_{\text {therm }}$ & $N_{T}$ & $T$ \\
\hline 4 & 50240 & 20000 & 19 & $0.50-1.40$ \\
5 & 50200 & 20000 & 19 & $0.50-1.40$ \\
6 & 50200 & 20000 & 19 & $0.50-1.40$ \\
7 & 50240 & 20000 & 19 & $0.50-1.40$ \\
8 & 102772 & 20000 & 19 & $0.50-1.40$ \\
9 & 51200 & 40000 & 19 & $0.50-1.40$ \\
10 & 51200 & 30000 & 19 & $0.50-1.40$ \\
12 & 40320 & 64000 & 19 & $0.50-1.40$ \\
15 & 5024 & 100000 & 49 & $0.55-1.40$ \\
16 & 10496 & 200000 & 33 & $0.60-1.40$ \\
\hline \hline
\end{tabular}

TABLE I: Simulation parameters for the bond-diluted $\pm J$ EA model. Number of samples $N_{s}$, minimal number of Monte Carlo sweeps for thermalization $N_{\text {therm }}$, the temperature range $T$, and the number of temperatures $N_{T}$ used for the PT as a function of the size $L$.

where the wave-vector-dependent SG susceptibility is defined by

$$
\chi_{\mathrm{SG}}(\mathbf{k})=\frac{1}{L^{3}} \sum_{\mathbf{x}} \sum_{\mathbf{r}} e^{i \mathbf{k r}}\left[\left\langle q_{\mathbf{x}} q_{\mathbf{x}+\mathbf{r}}\right\rangle_{\mathrm{T}}\right]_{\mathrm{av}}
$$

and where $\mathbf{k}_{\min }=(0,0,2 \pi / L)$ is the smallest nonzero wavevector allowed by periodic boundary conditions. The SG susceptibility is defined through $\chi_{\mathrm{SG}}(T, L)=$ $L^{3}\left\langle q^{2}\right\rangle=\chi_{\mathrm{SG}}(\mathbf{0})$. We denote the thermal average at temperature $T$ by $\langle\ldots\rangle_{\mathrm{T}}$ and the average over the disorder realizations by $[\ldots]_{\mathrm{av}}$. We also measure the Binder ratio defined by 36.37

$$
g(T, L)=\frac{1}{2}\left(3-\frac{\left[\left\langle q^{4}\right\rangle_{\mathrm{T}}\right]_{\mathrm{av}}}{\left[\left\langle q^{2}\right\rangle_{\mathrm{T}}\right]_{\mathrm{av}}^{2}}\right) .
$$

The parameters used for the simulation of the bonddiluted $\pm J$ EA model are given in Table I] whereas the ones used for the simulation of the site-diluted Gaussian EA model are given Table II] The number of replicas used at each temperature is two. The number of MC sweeps for the measurements is equal to the number of sweeps used for thermalization. Thermalization has been assured by requiring that the observables agree within errors in the last three bins of a logarithmic binning. The statistical errors of the MC observables are obtained from a jackknife estimate $\underline{38}$

\section{FSS ANALYSIS AND RESULTS}

In the following we make a detailed FSS analysis of the bond-diluted $\pm J$ EA model and present results on its critical behavior. After that we discuss a few results on the site-diluted Gaussian EA model which are mainly to motivate that in this model the finite-size effects are strong. Its FSS behavior is discussed in more detail in

\begin{tabular}{rrrrc}
\hline \hline$L$ & $N_{s}$ & $N_{\text {therm }}$ & $N_{T}$ & $T$ \\
\hline 4 & 8000 & 8000 & 21 & $0.40-1.40$ \\
6 & 8000 & 10000 & 21 & $0.40-1.40$ \\
8 & 8000 & 12000 & 21 & $0.40-1.40$ \\
10 & 4705 & 15000 & 21 & $0.40-1.40$ \\
12 & 4000 & 25000 & 21 & $0.40-1.40$ \\
16 & 3639 & 40000 & 21 & $0.40-1.40$ \\
20 & 333 & 90000 & 21 & $0.40-1.40$ \\
\hline \hline
\end{tabular}

TABLE II: Simulation parameters for the site-diluted Gaussian EA model. The meaning of the parameters is the same as in Table 1 .

Sec. VI] where we make a comparison between the FSS functions of the two models. An independent determination of the critical exponents of the site-diluted Gaussian EA model is not in the scope of this paper as the statistical quality of our data for this model is clearly poorer than the one of the data for the bond-diluted $\pm J \mathrm{EA}$ model, as can be seen from Tables \and II

\section{A. Bond-diluted $\pm J$ EA model}

In Fig. 2 we show the Binder ratio defined in Eq. (11) and the correlation length from Eq. (9) for the different system sizes used in the simulation (see Table I). The intersection of the curves for different system sizes defines an effective critical temperature that in the infinite volume limit converges to $T_{c}$. The evidence for a SG transition is very clear and a relatively precise and robust estimate of $T_{c} \approx 0.66$ can be obtained from this data. The intersection of the curves is very clean, especially also for the Binder ratio, where for other coupling distributions the crossing is not very clean. ${ }^{10,39}$ Moreover, a comparison with the results for the Binder ratio of the high-precision study of the undiluted $\pm J$ EA model in Ref. 10 gives evidence that the corrections to scaling for the bond-diluted $\pm J$ distribution used in our study are smaller, at least for this observable. The crossing is also very clean for the correlation length (apart from the data of the smallest lattice $L=4$ ), but this is less surprising as this quantity typically has relatively small corrections to scaling $\underline{\underline{10}}$

In Fig. 3 we show the data for the FSS functions $F_{\xi}$ and $F_{X S G}$ defined in Eq. (4). The FSS Ansatz works very well and we find very little differences between the data for the different lattice sizes used, indicating that the finitesize effects for these functions are already small. The data for the pair $L=4$ and $L=8$ (not shown in Fig. 3) on the other hand show noticeable finite-size corrections. In order to extrapolate the data for $\xi$ and $\chi_{\mathrm{SG}}$ to infinite volume we fit the data of $F_{\xi}$ and $F_{\chi_{\mathrm{SG}}}$ to the ansatz $F(x)=1+\sum_{i=1, n} a_{i} \exp (-i / x)$ with $n=5$. The fit is performed using the data of the largest system sizes, 


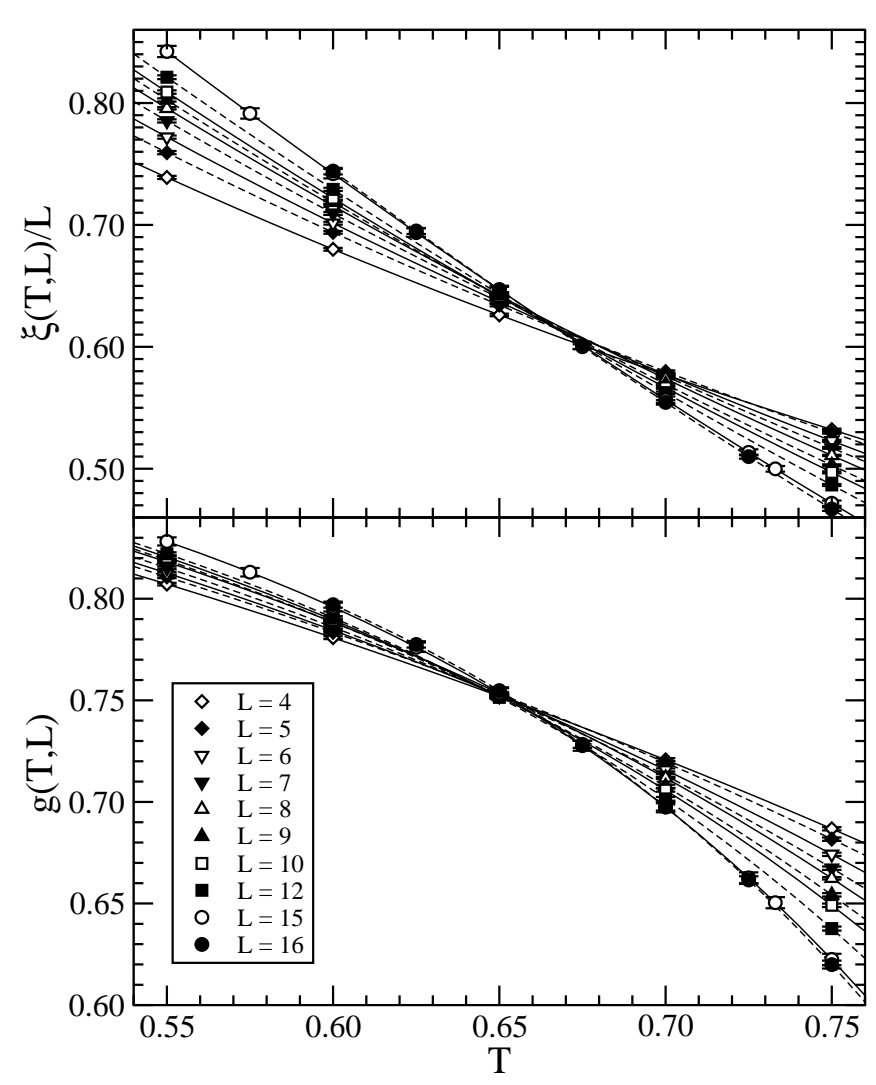

FIG. 2: The correlation length $\xi(T, L)$ measured in units of the lattice size $L$ and the Binder ratio $g(T, L)$ for the different system sizes in function of the temperature $T$. Note that the intersection of the different curves is very clean apart from that of the smallest system with $L=4$ and define $T_{c}$ with good precision. The crossings of $\xi(T, L) / L$ and $g(T, L)$ occur very close to each other which is an indication of small scaling violations. In order to make the inversion of the ordering below and above the phase transition more evident we have connected the data points with cubic splines.

i.e., the data of the pair $L=8$ and $L=16$. We found only slight changes using also the data from the smaller systems and the variations we get in the fitted quantities are well covered by the errors given in the final results. Using the iterative procedure described in Refs. 20 and 11 we obtain the infinite volume data $f_{\chi_{\mathrm{SG}}}$ which allows us to extract the ratio of the critical exponents $\gamma / \nu=$ $2-\eta=2.34 \pm 0.02$, as asymptotically $f_{\mathcal{O}} \sim x^{-x_{\mathcal{O}} / \nu}$ for $x \rightarrow \infty$, if $\mathcal{O}$ has a power-law divergence at $T_{c}$ with exponent $x_{\mathcal{O}}$ (see Fig. 7).

Following Ref. 11 we fit the data extrapolated to infi-

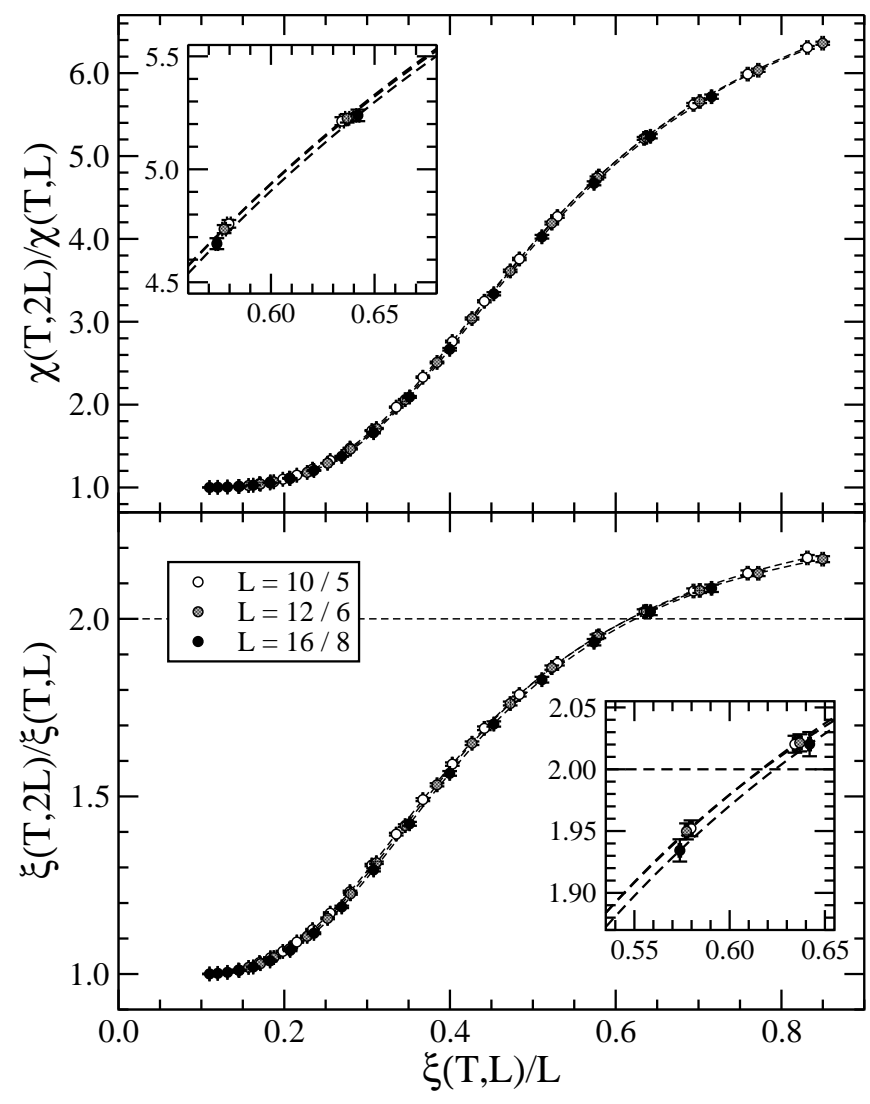

FIG. 3: The finite-size scaling functions $F_{\chi}$ and $F_{\xi}$ for the scale factor $s=2$ from which the critical exponents $\nu$ and $\eta$ are obtained. The insets show the same data around the effective critical point defined by $F_{\xi}=2$. The cubic splines connecting the data points show that the collapse of the data for the different sizes is very good. This is a clear sign of small scaling violations.

nite volume to

$$
\begin{gathered}
\xi(T)=c_{\xi}\left(T-T_{c}\right)^{-\nu}\left[1+\sum_{i=0}^{n} a_{\xi}^{(i)}\left(T-T_{c}\right)^{\theta^{(i)}}\right] \\
\chi_{\mathrm{SG}}(T)=c_{\chi}\left(T-T_{c}\right)^{-\gamma}\left[1+\sum_{i=0}^{n} a_{\chi}^{(i)}\left(T-T_{c}\right)^{\theta^{(i)}}\right]
\end{gathered}
$$

as well as

$$
\chi_{\mathrm{SG}}(\xi)=b \xi^{2-\eta}\left[1+\sum_{i=0}^{n} d^{(i)} \xi^{-\Delta^{(i)}}\right],
$$

with the correction-to-scaling exponents $\theta^{(i)}$ and $\Delta^{(i)}$. In particular we have $\theta^{(0)}=\omega \nu$ and $\Delta^{(0)}=\omega$. We find that including a nonleading correction term for the fits of $\chi_{\mathrm{SG}}$ (i.e., $n=1$ in Eqs. (13) and (14)) reduces the dependence on the fitting range considerably, whereas for the fits of $\xi$ the leading correction is enough for obtaining stable and 
good fits (i.e., $n=0$ in Eq. (12)). We obtain as preferred values

$$
\begin{gathered}
\nu=2.17(15), \quad \eta=-0.336(20), \quad \gamma=4.96(30), \\
T_{c}=0.663(6) \quad \text { and } \omega=0.7(3) .
\end{gathered}
$$

Note that the values we obtain for $\gamma, \nu$ and $\eta$ are well compatible with the scaling relation $\gamma=\nu(2-\eta)$. The value for the correction-to-scaling exponent $\omega$ has to be taken as an effective value as we do not separate possible analytical from nonanalytical corrections to scaling in our fits (see Ref. 18 for a discussion).

Using on the other hand the quotient method with Eqs. (6) and (7) again for the data of the pair $L=8$ and $L=16$ we obtain

$$
\begin{gathered}
\nu=2.22(15), \quad \eta=-0.349(18), \\
\xi\left(T_{c}, L\right) / L=0.625(10) .
\end{gathered}
$$

In order to fine tune the ratio $\xi / L$ on the two lattices to be equal and in order to determine the derivative in Eq. (7) we use a cubic spline fit to the data. The use of the cubic spline fits and the remaining scaling violations are the main sources of systematic errors. We consider them, however, to be smaller than the statistical errors in the determination of the universal FSS functions even for the rather large number of coupling realizations we use in this study.

Fig. 4 shows the FSS function $F_{g}$ of the Binder ratio $g$. The value of $\xi\left(T_{c}^{*}, L\right) / L$ at which $F_{g}=1$ gives an alternative possibility to define an effective critical point. The fact that $\xi\left(T_{c}^{*}, L\right) / L$ defined from $F_{g}=1$ (shown in the inset of Fig. (4) is slightly larger than the one defined from $F_{\xi}=2$ (see inset of Fig. 3) indicates that there are remaining corrections to scaling. The value of $\xi\left(T_{c}^{*}, L\right) / L$ taken from the pair $L=8$ and $L=16$ and the condition $F_{g}=1$ is $\xi\left(T_{c}^{*}, L\right) / L=0.635(15)$. Comparing to the corresponding value from the pair $L=8$ and $L=16$ and the condition $F_{\xi}=2$, which is $\xi\left(T_{c}^{*}, L\right) / L=0.625(10)$, we can conclude that these remaining corrections to scaling are small, however.

In Fig. 5 we show the universal FSS function defined by the Binder ratio $g(T, L)$ as a function of the correlation length $\xi(T, L) / L$. This FSS function is particularly interesting as these two dimensionless quantities have a rather large cross correlation. This means that all the data for the sizes from $L=8$ to 16 fall on the same curve within their statistical errors. The curve provides a oneto-one correspondence between the two quantities that are often used to determine the critical point in SG simulations. Using the value $\xi\left(T_{c}, L\right) / L=0.625(10)$ from the condition $F_{\xi}=2$ as our most reliable determination of this quantity we obtain a corresponding value of the Binder ratio at the critical point of $g\left(T_{c}\right)=0.742(7)$.

\section{B. Site-diluted Gaussian EA model}

In Fig. 6 we show the Binder ratio defined in Eq. (11) and the correlation length from Eq. (9) for the different

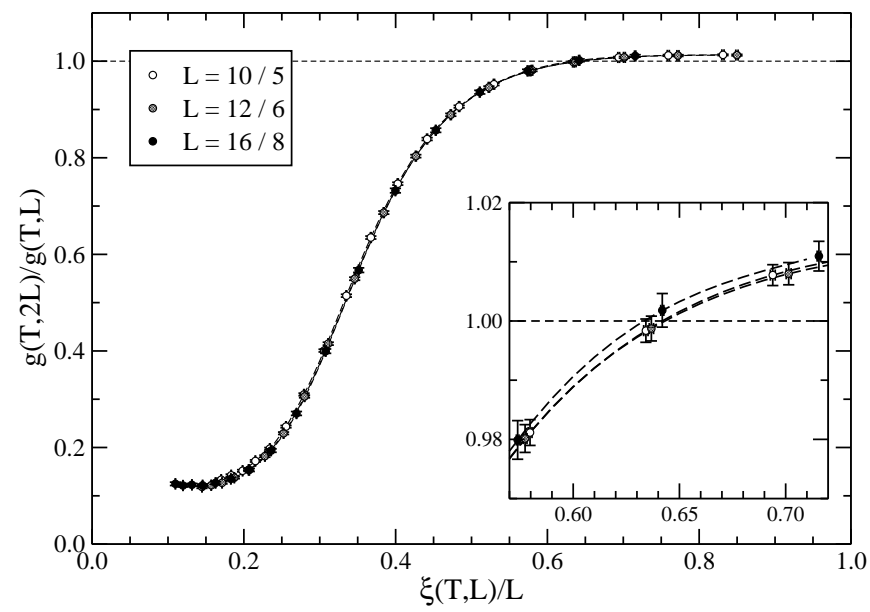

FIG. 4: The FSS function of the Binder ratio $g(T, L)$. The inset shows the same data around the effective critical point defined by $F_{g}=1$. The broken lines connecting the data points are cubic splines making it again evident that scaling violations are small.

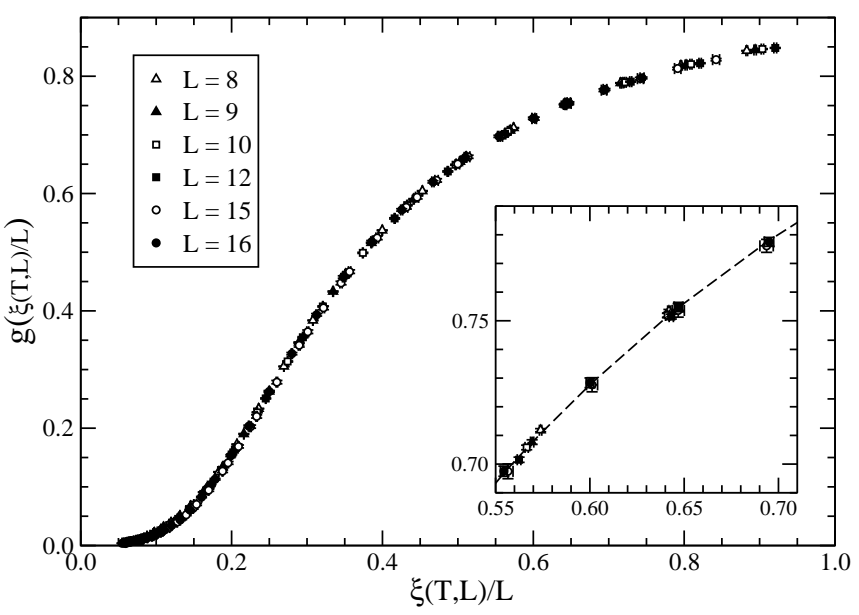

FIG. 5: The universal FSS function defined by the Binder ratio $g(T, L)$ versus the correlation length $\xi(T, L) / L$. Note that due to the very small scaling violations the plot defines a precise mapping between the two quantities that are typically used to define the critical temperature. The inset shows the same data around the effective critical point. The broken line is a polynomial fit through all the data points (i.e., $L=$ $8, \ldots, 16)$ in the range $0.5 \leq \xi(T, L) / L \leq 0.8$.

system sizes used in the simulation (see Table II). In contrast to the data for the bond-diluted $\pm J$ model shown in Fig. 2 the crossing of the curves for the correlation length is no longer very neat. Moreover, the Binder ratio does not cross at all for the system sizes and the temperature range used in this simulation. With increasing system sizes the crossing of the correlation length shifts from larger $T$ values toward $T \approx 0.51$. Although there is no crossing of the Binder ratio we consider the data of the correlation length to be clear enough evidence for a $\mathrm{SG}$ transition around $T_{c} \approx 0.51$. We attribute the fact 


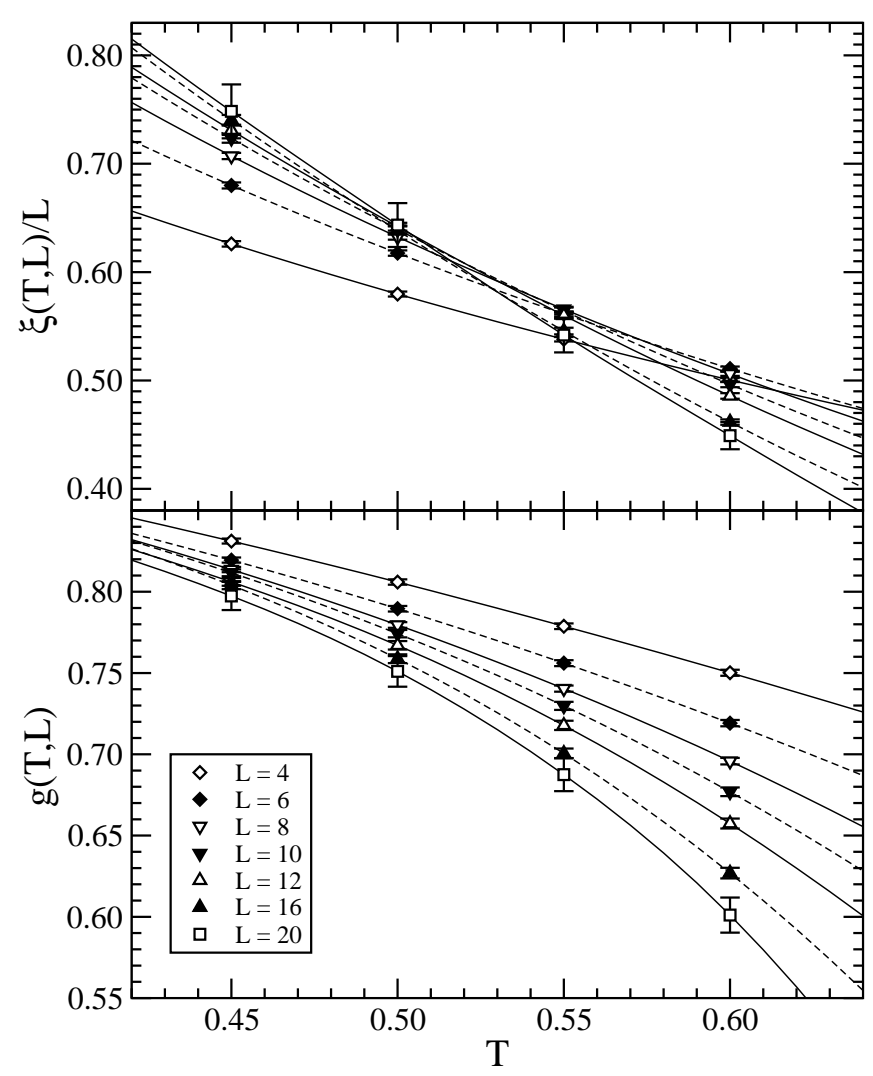

FIG. 6: The same as Fig. 2, but for the site-diluted Gaussian coupling distribution. The correlation length shows a crossing at $T_{c} \approx 0.51$. The large shift between the crossing points for the smaller system sizes indicates rather large finite-size corrections. These large scaling corrections can be seen even better for the Binder ratio, as it does not even cross for the different system sizes and temperature range used in this simulation. The data points are connected by cubic splines.

that there is no clean intersection to the presence of very strong corrections to scaling in this model and we will give evidence for this view in Sec. VI] We think that the corrections are so large that nonleading correction terms cannot be neglected.

\section{UNIVERSALITY OF THE FSS FUNCTIONS}

It is obviously interesting to see how the FSS functions of these two coupling distributions having such a different level of scaling corrections compare to each other and that is what we are going to do in this section. But first we compare the FSS functions of the bond-diluted $\pm J$ EA model with the ones of the undiluted $\pm J$ EA model.

In Fig. 7 we compare the data for the FSS functions $f_{\xi}$ and $f_{\chi_{\mathrm{SG}}}$ defined in Eq. (3) obtained in this simulation with the data from the $3 \mathrm{D}$ undiluted $\pm J$ EA model obtained by Palassini and Caracciolo in Ref. 11. The agreement of the two data sets is excellent giving very strong evidence that above the critical dilution $p^{*}$ the

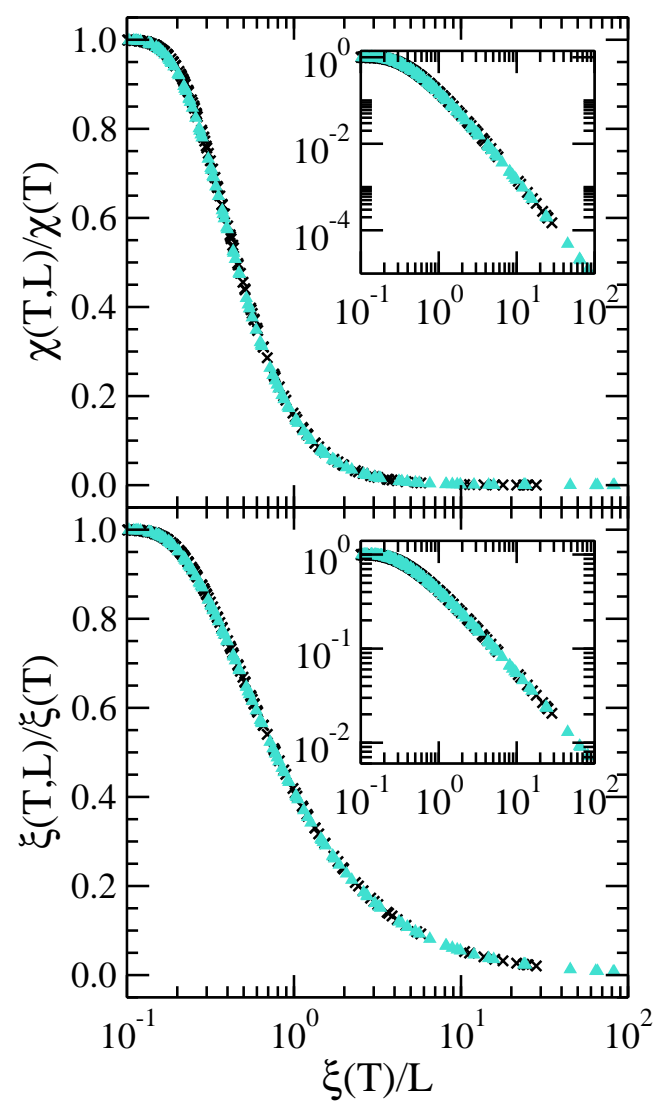

FIG. 7: (Color online) A comparison of the universal FSS functions $f_{\chi}$ and $f_{\xi}$ for the scale factor $s=2$ of the bonddiluted $\pm J$ EA model (circles, $L=4$ to $L=16$ ) with the corresponding data for the undiluted $\pm J$ EA model from Palassini and Caracciolo 11 (triangles, $L=5$ to $L=48$ ). The insets show the same data in a log-log plot, making evident the power-law decay at large $\xi(T, \infty) / L$. The excellent agreement between the two data sets gives strong evidence that above the critical dilution $p^{*}$ the critical behavior of the 3D $\pm J$ EA model does not depend on bond dilution.

critical behavior of the $\pm J$ EA model does not depend on bond dilution.

In Fig. 8 we compare the data for the FSS function defined by the Binder ratio $g(T, L)$ versus the correlation length $\xi(T, L) / L$ for the bond-diluted $\pm J$ with the site-diluted Gaussian coupling distribution. In Fig. 5 we showed that this function shows very little scaling corrections for the bond-diluted $\pm J$ EA model. Not surprisingly this is not the case for the site-diluted Gaussian EA model. The curves for increasing system sizes are, however, moving closer and closer to the curve defined by the data of the bond-diluted $\pm J$ EA model. The agreement between the curves for the largest few system sizes is very good giving support to the claim that the two models fall into the same universality class.

In Fig. 9 we compare the data for the FSS functions $F_{\xi}$ and $F_{\chi_{\mathrm{SG}}}$ as defined in Eq. (4) of the bond-diluted $\pm J \mathrm{EA}$ model with corresponding ones of the site-diluted Gaussian EA model for the pair $L=8$ and $L=16$. The 


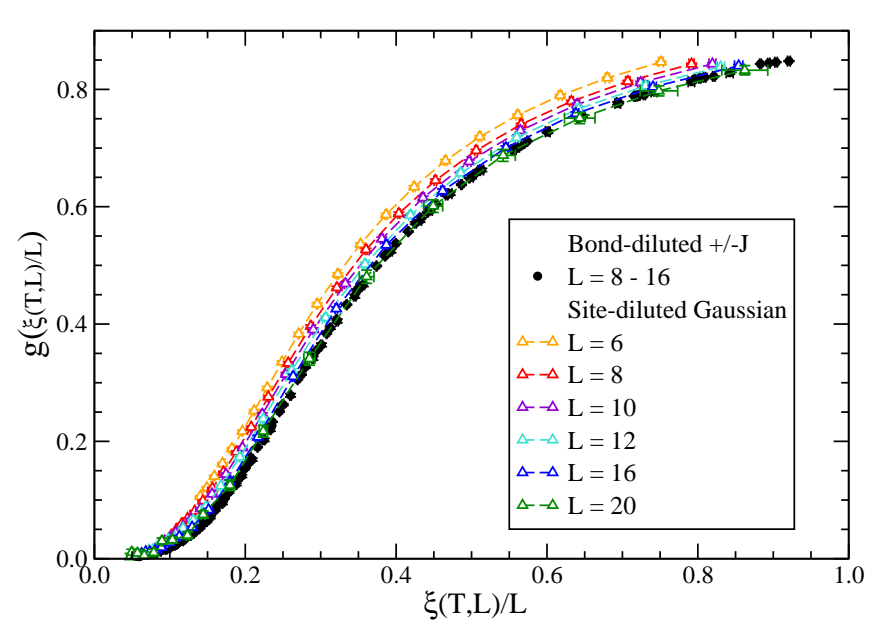

FIG. 8: (Color online) A comparison of the universal FSS function defined by the Binder ratio $g(T, L)$ versus the correlation length $\xi(T, L) / L$ (see also Fig. [5) is given for the bond-diluted $\pm J$ and the site-diluted Gaussian EA model. The figure shows how the data of the site-diluted Gaussian model with increasing system size converges toward the curve defined by the data of the bond-diluted $\pm J$ model. The data for the site-diluted Gaussian EA model is connected by cubic splines to make the convergence more evident.

agreement is again very good. Without performing any calculation we can read off this figure that the critical exponents $\eta$ and $\nu$ of the two models are fully compatible with each other within errors.

\section{DISCUSSION AND CONCLUSIONS}

Replica cluster MC algorithms $\frac{3,4,5,6}{3}$ do not only allow for an efficient simulation of the two-dimensional EA model, but also for different 3D diluted models with disorder and frustration. ${ }^{7}$ We have applied a variant of such an algorithm to the simulation of the $3 \mathrm{D}$ bond-diluted EA model with binary couplings. The universal FSS functions are determined with very high statistical accuracy and they show little size dependence indicating that the corresponding finite-size corrections are becoming small when determined from a pair of systems with $L=5$ and $L=10$ or larger. Clearly a change of the behavior of the FSS functions at larger sizes than considered here cannot completely be excluded, but seems rather improbable. In order to obtain good knowledge on the magnitude of the remaining scaling corrections a more precise study with even more statistics would be needed. Extrapolating the data for the correlation length $\xi$ and the SG susceptibility $\chi_{\mathrm{SG}}$ to infinite volume we obtain the critical exponents $\nu=2.17(15), \eta=-0.336(20)$, and the effective leading correction-to-scaling exponent $\omega=0.7(3)$. The value of $\gamma=4.96(30)$ that we obtain is well compatible with values of $\nu, \eta$ and the scaling relation $\gamma=\nu(2-\eta)$ connecting these exponents. For the value of the critical temperature we get $T_{c}=0.663(6)$. On the other

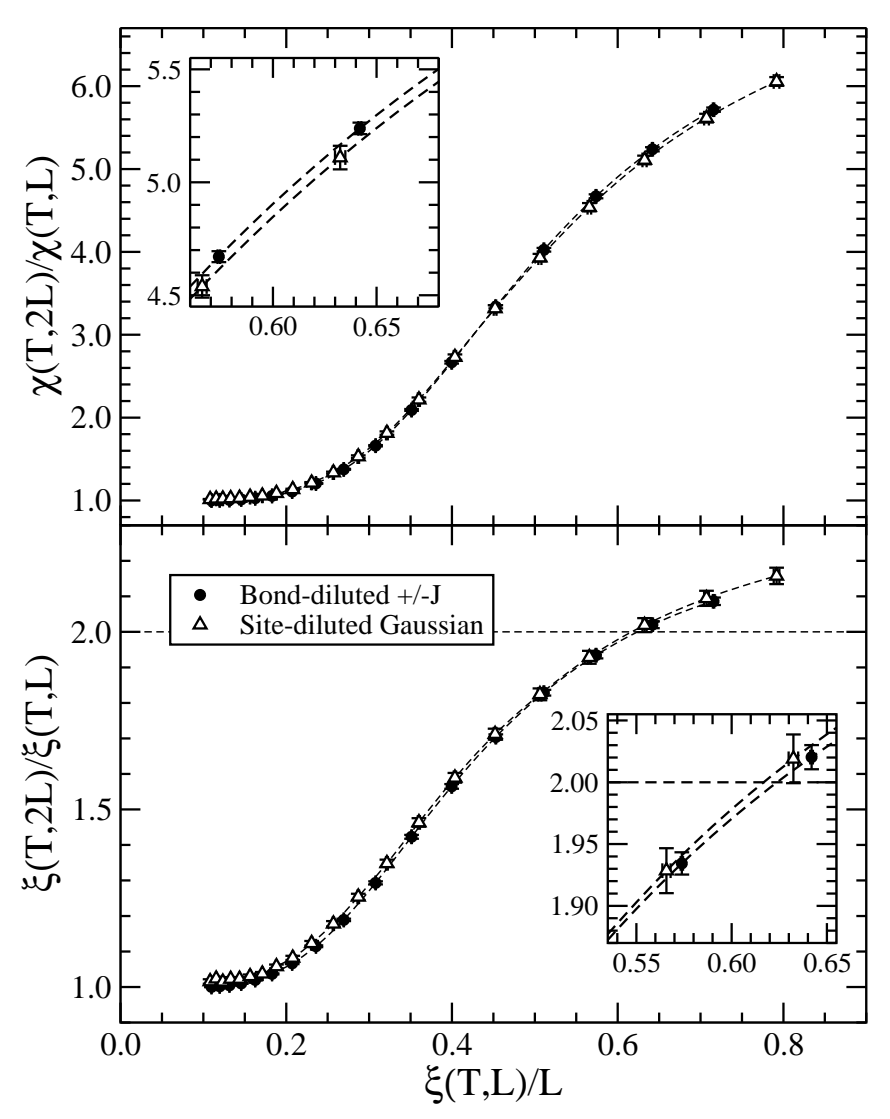

FIG. 9: A comparison of the finite-size scaling functions $F_{\chi}$ and $F_{\xi}$ for the scale factor $s=2$ is given for the bond-diluted $\pm J$ and the site-diluted Gaussian EA model for the pair $L=8$ and $L=16$. The insets show the same data around the effective critical point defined by $F_{\xi}=2$. From the fact that the data collapse is very good we can conclude that the estimates for the critical exponents $\nu$ and $\eta$ for the two models are the same within errors.

hand using the quotient method we extract $\nu=2.22(15)$ and $\eta=-0.349(18)$. Other critical quantities we obtain from this method are $\xi\left(T_{c}, L\right) / L=0.625(10)$ and using the one-to-one correspondence between the Binder ratio and the correlation length (see Fig. (5) we also have $g\left(T_{c}\right)=0.742(7)$. We slightly prefer the values of the critical exponents obtained by the quotient method over those obtained by the extrapolation to infinite volume technique because of two reasons. The first being that the fits are clearly more robust using the quotient method and the second being that the exponents are extracted at the apparent critical point $T_{c}^{*}$ which allows for a more precise numerical determination of their values $\stackrel{10,19}{\underline{1}}$ Therefore we quote as final values for the critical exponents of the $3 \mathrm{D}$ bond-diluted $\pm J$ EA model $\nu=2.22(15)$ and $\eta=-0.349(18)$.

The comparison of the universal FSS functions $f_{\xi}$ and $f_{\chi_{\mathrm{SG}}}$ with the ones from the $3 \mathrm{D}$ undiluted $\pm J$ EA model obtained by Palassini and Caracciolo in Ref. 11 shows perfect agreement. This is strong evidence that bond dilution is not a relevant perturbation, i.e., it does not 
change the critical behavior of the standard $3 \mathrm{D} \pm J$ EA model in a large range of dilutions and most probably down to the critical dilution $p^{*}$ at which the SG phase appears. This conclusion is furthermore supported by the excellent agreement between the critical values obtained in this study $[\nu=2.22(15), \eta=-0.349(18)]$ with the ones from the high-precision study in Ref. 10, where $\nu=$ $2.15(15)$ and $\eta=-0.337(15)$ are given.

At this point a remark on the difference between the critical exponents obtained by Palassini and Caracciolo 11 is in order, since they give $\nu=1.8(2)$ and $\eta=-0.26(4)$ using the same infinite volume extrapolation technique used in this paper and moreover as they have (within errors) the same universal FSS functions $f_{\xi}$ and $f_{\chi_{S G}}$ as we have obtained in this paper. We think that this discrepancy is due to the fact that a precise determination of the critical exponents needs data of very high accuracy close to the effective critical point. This was not the case in the Palassini and Caracciolo $\underline{11}$ study that involved mainly data from the paramagnetic region and therefore they were unable to constrain the fits enough to provide exact and robust values for the critical exponents.

The additional comparison of the universal FSS functions $F_{\xi}$ and $F_{\chi \mathrm{SG}}$ of the bond-diluted $\pm J$ coupling distribution with the corresponding ones of the site-diluted Gaussian coupling distribution shows that claims for the nonuniversality of the 3D EA model using dynamical MC $\underline{12,13,14,16}$ are clearly not supported by a careful static $\mathrm{MC}$ analysis. The agreement of the FSS functions is very good and excludes large differences between the critical exponents of the two models. Smaller differences at the level of $10 \%$ obviously cannot be excluded completely with the given precision of this study, but looking at the complete behavior of the FSS functions (and not only at the critical point) the agreement seems too good to leave much space for nonuniversal critical behavior of the 3D EA model. This is further supported by the agreement between the two models regarding the FSS function defined by the Binder ratio versus the correlation length. In the case of the site-diluted Gaussian EA model this FSS function clearly is showing large corrections to scaling for smaller system sizes that, however, asymptotically vanish leading most probably to a unique, i.e., universal limiting FSS function. We conclude that from static MC simulations, especially from the comparison of different FSS functions, we get strong evidence for universal critical behavior of the SG transition in the 3D EA model. The conclusion that the critical behavior of the 3D EA model is universal is also reached in Ref. 17. There the authors perform a high-precision MC simulation to study the static properties of the model using mainly a Gaussian and $a \pm J$ distribution for the couplings. It has to be noted that the values of the critical exponents they find, especially the one for $\eta$ of the $\pm J$ distribution, is not fully consistent with the ones of the present study as they find $\nu=2.44(9)$ and $\eta=-0.37(5)$ for the Gaussian as well as $\nu=2.39(5)$ and $\eta=-0.395(17)$ for the $\pm J$ distribution. We consider this difference to be due to the different techniques to extract the critical exponents used in their paper, which especially for the value of $\eta$ is rather sensitive to nonanalytical as well as analytical corrections to scaling. In Ref. 40 the $3 \mathrm{D} \pm J$ EA model is studied on very large lattices with a new technique to extract critical exponents. The critical exponents obtained in this study are $\nu=2.72(8)$ and $\eta=-0.4(4)$ and differ again from the ones of our study and also the ones of Ref. 17. We think that the spread in the above results reflects the difficulties to extract critical quantities in the 3D EA model with high precision and in this respect we consider the comparison of the complete FSS functions a more reliable and moreover parameter-free tool to check for universality.

Finally, we have noted that the observables we have studied in this paper show rather little scaling corrections for the $3 \mathrm{D}$ bond-diluted $\pm J$ EA model at the given bond occupation of $p=0.45$. This is clearly in accordance with the findings of the high-temperature series study in Ref. 8 and the ground state defect energies study in Ref. 9 . This important physical aspect together with all the technical advantages of the replica cluster MC algorithm and the possibility of multispin-coding makes this model clearly a very interesting candidate for further studies of the static properties of spin glasses.

\section{Acknowledgments}

It is a pleasure to thank M. Palassini, F. RicciTersenghi, G. Parisi, H. G. Katzgraber, A. P. Young, and F. Niedermayer for interesting discussions. The simulations have been mainly performed on the workstations of the institute of theoretical physics in Bern and in parts on the CLX cluster of the CINECA and the clusters IDRA and PIOVRA in Roma. This work was supported by EEC's HPP under contract No. HPRN-CT-2002-00307 (DYGLAGEMEM) and ECC's FP6 IST Programme under contract No. IST-001935 (EVERGROW).
1 S. F. Edwards and P. W. Anderson, Theory of spin glasses, J. Phys. F: Met. Phys. 5, 965 (1975).

2 H. T. Diep, ed., Frustrated Spin Systems (World Scientific, 2005), chap. 9.

${ }^{3}$ R. H. Swendsen and J.-S. Wang, Replica Monte Carlo sim- ulation of spin-glasses, Phys. Rev. Lett. 57, 2607 (1986).

${ }^{4}$ O. Redner, J. Machta, and L. F. Chayes, Graphical representations and cluster algorithms for critical points with fields, Phys. Rev. E 58, 2749 (1998).

5 J. Houdayer, A cluster Monte Carlo algorithm for 2- 
dimensional spin glasses, Eur. Phys. J. B. 22, 479 (2001).

6 J.-S. Wang and R. H. Swendsen, Replica Monte Carlo simulation (Revisited), Prog. Theor. Phys. Suppl. 157, 317 (2005)

7 T. Jörg, Cluster Monte Carlo algorithms for diluted spin glasses, Prog. Theor. Phys. Suppl. 157, 349 (2005).

8 S. Shapira, L. Klein, J. Adler, A. Aharony, and A. B. Harris, Phase diagram of the dilute Ising spin glass in general spatial dimension, Phys. Rev. B 49, 8830 (1994).

9 S. Boettcher, Stiffness exponents for lattice spin glasses in dimensions $d=3, \ldots, 6$, Eur. Phys. J. B 38, 83 (2004).

${ }^{10}$ H. G. Ballesteros, A. Cruz, L. A. Fernández, V. MartínMayor, J. Pech, J. J. Ruiz-Lorenzo, A. Tarancón, P. Téllez, C. L. Ullod, and C. Ungil, Critical behavior of the threedimensional Ising spin glass, Phys. Rev. B 62, 14237 (2000).

11 M. Palassini and S. Caracciolo, Universal Finite-Size Scaling Functions in the 3D Ising Spin Glass, Phys. Rev. Lett. 82, 5128 (1999).

12 L. W. Bernardi, S. Prakash, and I. A. Campbell, Ordering Temperatures and Critical Exponents in Ising Spin Glasses, Phys. Rev. Lett. 77, 2798 (1996).

13 L. W. Bernardi and I. A. Campbell, Critical exponents in Ising spin glasses, Phys. Rev. B 56, 5271 (1997).

14 P. O. Mari and I. A. Campbell, The ordering temperature and critical exponents of the bimodal Ising spin glass in dimension three, Phys. Rev. B 65, 184409 (2002).

15 D. Daboul, I. Chang, and A. Aharony, Test of universality in the Ising spin glass using high temperature graph expansion, Eur. Phys. J. B 41, 231 (2004).

16 M. Pleimling and I. A. Campbell, Dynamic critical behavior in Ising spin glasses, Phys. Rev. B 72, 184429 (2005).

17 H. G. Katzgraber, M. Körner, and A. P. Young, Universality in three-dimensional Ising spin glasses: A Monte Carlo study, Phys. Rev. B 73, 224432 (2006).

18 F. Parisen Toldin, A. Pelissetto, and E. Vicari, Critical behaviour of the random-anisotropy model in the stronganisotropy limit, J. Stat. Mech. 2006, P06002 (2006).

19 H. G. Ballesteros, L. A. Fernández, V. Martín-Mayor, and A. Muñoz Sudupe, Critical properties of the Antiferromagnetic $R P^{2}$ model in three dimensions, Nucl. Phys. B 483, 707 (1997).

20 S. Caracciolo, R. G. Edwards, S. J. Ferreira, A. Pelissetto, and A. D. Sokal, Extrapolating Monte Carlo Simulations to Infinite Volume: Finite-Size Scaling at $\xi / L \gg 1$, Phys. Rev. Lett. 74, 2969 (1995).

21 A. B. Harris, Effect of random defects on the critical behaviour of Ising models, J. Phys. C 7, 1671 (1974).

22 G. Parisi, F. Ricci-Tersenghi, and J. J. Ruiz-Lorenzo, Universality in the off-equilibrium critical dynamics of the $3 d$ diluted ising model, Phys. Rev. E 60, 5198 (1999).
23 S. Jiménez and T. Jörg, in preparation.

24 A. J. Bray and S. Feng, Percolation of order in frustrated systems: The dilute J spin glass, Phys. Rev. B 36, 8456 (1987).

25 M. E. Fisher, in Proceedings of the 51st Enrico Fermi Summer School, edited by M. S. Green (Academic Press, New York, 1972).

26 V. Privman, ed., Finite Size Scaling and Numerical Simulation of Statistical Systems (World Scientific, Singapore, 1990).

27 R. H. Swendsen and J.-S. Wang, Nonuniversal critical dynamics in Monte Carlo simulations, Phys. Rev. Lett. 58, 86 (1987).

28 H. G. Ballesteros, L. A. Fernández, V. Martín-Mayor, A. Muñoz Sudupe, G. Parisi, and J. J. Ruiz-Lorenzo, Critical exponents of the three-dimensional diluted Ising model, Phys. Rev. B 58, 2740 (1998).

${ }^{29}$ K. Hukushima and K. Nemoto, Exchange Monte Carlo method and application to spin glass simulations, J. Phys. Soc. Jpn. 65, 1604 (1996).

30 L. Viana and A. J. Bray, Phase diagrams for dilute spin glasses, J. Phys. C 18, 3037 (1985).

31 N. Persky, I. Kanter, and S. Solomon, Cluster dynamics for randomly frustrated systems with finite connectivity, Phys. Rev. E 53, 1212 (1996).

32 F. Niedermayer, General Cluster Updating Method for Monte Carlo Simulations, Phys. Rev. Lett. 61, 2026 (1988).

33 J. Hoshen and R. Kopelman, Percolation and cluster distribution. I. Cluster multiple labeling technique and critical concentration algorithm, Phys. Rev. B 14, 3438 (1976).

${ }^{34}$ F. Cooper, B. Freedman, and D. Preston, Solving $\phi_{1,2}^{4}$ theory with Monte Carlo, Nucl. Phys. B 210, 210 (1982).

35 J. K. Kim, Asymptotic scaling of the mass gap in the twodimensional $O(3)$ nonlinear sigma model: A numerical study, Phys. Rev. D 50, 4663 (1994).

36 K. Binder, Critical properties from Monte Carlo coarse graining and renormalization, Phys. Rev. Lett. 47, 693 (1981).

37 K. Binder, Finite size scaling analysis of Ising model block distribution functions, Z. Phys. B 43, 119 (1981).

38 B. Efron, The Jackknife, the Bootstrap, and Other Resampling Plans (SIAM, 1982).

39 N. Kawashima and A. P. Young, Phase transition in the three-dimensional $\pm J$ Ising spin glass, Phys. Rev. B 53, R484 (1996).

40 I. A. Campbell, K. Hukushima, and H. Takayama, Extended scaling scheme for critically divergent quantities in ferromagnets and spin glasses, Phys. Rev. Lett. 97, 117202 (2006). 